\title{
Review of: "Multicentric real world evidence with palbociclib in hormone positive HER2 negative metastatic breast cancer in Indian population"
}

\author{
Didier Verhoeven ${ }^{1}$
}

1 AZ KLINA

Potential competing interests: The author(s) declared that no potential competing interests exist.

Review: Multicentric real world evidence with palbocyclib in hormono positive HER2 negative metastatic breast cancer in Indian population. (Scientific Reports , Aug 10. 2021)

August 2021 real world data on the use of palbocyclib in the Indian population were published, suggesting effectiveness similar to what was previously published (1). Although these data were interesting and reassuring some remarks must be made. In addition I took the opportunity to comment on global quality care for breast cancer.

This study was reported to be the largest multi-centric study in the Indian population. Nevertheless only 188 patients from only two centers in New Delhi could be included during a period of three years. To be conclusive for a country of more than 1 billion inhabitants it remains small. Nevertheless the data from Asian patients are important to demonstrate the usefulness in this population. An anthropological approach is indeed needed to understand how possible differences in breast cancer genetics in a wider social and cultural context can impact the tumor responses in different parts of the world (2). Not only social inequalities in access to technologies and treatment are important to understand differences but also ethnographic variations and population genetics. In the US for example five different populations with different mortality risk were defined: White, Afro-Americans, Hispanic, Asian-Pacific Islander and IndianAlaska native. Recent SEER data analysis showed very different mortality rates for these populations, respectively: 156, 177, 110, 97 and 141. In the Afro-American population not only socio-economic differences were observed but also more aggressive and biologic different tumors.

In this retrospective study the routine evaluation with the costly CT and PET-CT scan can be questioned especially in LMIC. Although the dose of $250 \mathrm{mg}$ fulvestrant can be defended in a LMIC, we have to stress that the most efficient standard dose is $500 \mathrm{mg} \mathrm{(3).}$

An additional and important finding in this study is the high number of young, predominantly premenopausal (57\%) patients with a late presentation of their disease.

As breast cancer death disproportionately affect women in low- and middle-income countries, the time is now to engage global partners to coordinate sustainable efforts and achieve favorable outcomes for these patients. Therefore, the WHO launched the Global Breast Cancer Initiative that focuses on three main 
aspects of breast cancer care: health promotion, timely diagnosis and comprehensive treatment. During the last 10 years we devoted much efforts to promote a fairer breast cancer care in a globalized world (4). An executive summary and consensus had already been published by Anderson et al. in the Lancet Oncology (5). It is fundamental to make breast cancer care a public health priority for all women worldwide by establishing resource-stratified guidelines (www.bhgi.info). An audiogram discussing our work is freely available at https://research.one.surgery/new-frontiers-for-fairer-breast-cancer-care-in-aglobalized-world/ Another aspect is the discussion about availability of essential medicines worldwide. Since 1977 the Essential Medicine List is updated by the WHO on a two-yearly basis (6). Medicines key to improve survival and/or better Quality of Life are listed, embodying the concept that some medicines are more important than others based on relative efficacy and/or safety. Currently the 21ste list is used, lastly updated june 2019 containing 59 immunomodulators and antineoplastics for malignant diseases. The list can be used by countries to help developing their own priorities. Although their importance for breast health CDK4/6 inhibitors and Fulvestrant are not included. Cancer medicine availability and shortage, out-of-pocket costs and accessibility remains a high priority not only in developing countries but also in Europe.

\section{References}

1. Agrawal et al. Multicentric real world evidence with palbocyclib in hormone positive HER2 negative metastatic breast cancer in Indian population Scientific Reports (2021) 11: 16236

2. Gibbon S. Translating population differences : The use and re-use of genetic ancestry in Brazilian Cancer Genetics. Medical Anthropology (2016) 35:1, 58-72

3. Di Leo et al. Confirm: A phase III randomized, parallel-group trial comparing fulvestrant $250 \mathrm{mg}$ vs fulvestrant $500 \mathrm{mg}$ in post-menopausal women with estrogen receptor-positive advanced breast cancer. JCO (2010 ) 28: 4594-4600

4. Didier Verhoeven, Cary Kaufman, Robert Mansel, Sabine Siesling, Editors, Breast cancer: Global Quality Care (2020) , Oxford University Press

5. Anderson BO et al. Optimisation of Breast Cancer Management in low-resource and middle-resource countries: executive summary of the Breast Health Global Initiative consensus (2010) Lancet Oncol (2011) 12:387-398

6. World Health Organization (WHO) Model Lists of Essential Medicines 2019 Available from :http://www.who.int/medicines/publications/essentialmedicines/en/ 